\title{
Chronic Placental Inflammation as a Risk Factor of Severe Retinopathy of Prematurity
}

Chae Young Kim · Euiseok Jung Eun $\mathrm{Na} \mathrm{Kim}^{1}$. Chong Jai Kim ${ }^{1}$ Joo Yong Lee ${ }^{2} \cdot$ Ji Hye Hwang Woo Sun Song · Byong Sop Lee Ellen Ai-Rhan Kim · Ki-Soo Kim

Departments of Pediatrics, ${ }^{1}$ Pathology, and ${ }^{2}$ Ophthalmology, Asan Medical Center, University of Ulsan College of Medicine, Seoul, Korea

Received: May 10, 2018

Revised: June 26, 2018

Accepted: July 9, 2018

\section{Corresponding Author}

Euiseok Jung, MD

Department of Pediatrics, Asan Medical Center,

University of Ulsan College of Medicine,

88 Olympic-ro 43-gil, Songpa-gu, Seoul 05505,

Korea

Tel: +82-2-3010-3372

Fax: +82-2-3010-7302

E-mail: euisjung@amc.seoul.kr

\begin{abstract}
Background: Chronic placental inflammation (CPI) has been implicated in the pathogenesis of diseases in premature infants, whereas retinopathy of prematurity (ROP) is a major complication primarily affecting preterm and very low-birth-weight (VLBW) infants. This study aims to investigate the association between CPI and ROP in VLBW infants. Methods: We performed a retrospective review of clinical records of VLBW infants born between 2013 and 2016. Placental pathology findings including CPI cases were analyzed using logistic regression to study infants' morbidities and other clinical characteristics. Results: A total of 402 infants with a mean (standard deviation) gestational age of $28.5(2.8)$ weeks and birth weight of 1,027.2 (304.4) g were included. The incidence of ROP was $24.1 \%$. CPI was found in 90 infants (22.4\%), among which $28.9 \%$ (26 of 90 ) developed ROP, and $21.1 \%$ (19 of 90) underwent laser photocoagulation. Lower gestational age, lower birth weight, longer duration of oxygen supply, and presence of CPI were associated with the development of ROP. After adjustment for gestational age, birth weight, sex, duration of oxygen supply, and other overlapping placental pathology, CPI was associated with the odds for type 1 ROP that required laser photocoagulation (adjusted odds ratio, 2.739; 95\% confidence interval, 1.112 to $6.749 ; p=.029$ ). Conclusions: $C P I$ was associated with severe ROP requiring treatment with laser photocoagulation in VLBW infants.
\end{abstract}

Key Words: Chronic chorioamnionitis; Villitis; Deciduitis; Retinopathy of prematurity; Infant, very low birth weight
Preterm birth is associated with an increased risk of perinatal mortality and long-term morbidity. ${ }^{1}$ The pathological findings of the placenta often provide valuable information regarding the clinical outcomes of preterm infants because the changes in the placenta mirror intrauterine environments affecting the fetus. ${ }^{2,3}$ Acute and chronic placental inflammations are common pathology of preterm birth and are significantly associated with preterm birth and neonatal morbidity. ${ }^{1-4}$ While acute placental inflammation (API) (acute chorioamnionitis and acute funisitis) is caused by the infiltration of neutrophils into the placental compartments, chronic placental inflammation (CPI) (chronic chorioamnionitis, chronic villitis, and chronic deciduitis) is characterized by the infiltration of lymphocytes, plasma cells, and/or macrophages. ${ }^{5,6}$ API accompanies elevation of proinflammatory cytokines such as interleukin (IL)-6 and IL-8 in the amniotic fluid and fetal plasma. On the other hand, CPI is associated with elevation of antiangiogenic T-cell chemokines CXCL9, CXCL10, and CXCL11 in the amniotic fluid and fetal plasma. ${ }^{6}$ Therefore, it is very plausible that API and CPI affect the systemic biology of preterm newborns.

Illnesses associated with prematurity include respiratory distress syndrome (RDS), intraventricular hemorrhage (IVH), bronchopulmonary dysplasia (BPD), and retinopathy of prematurity (ROP). The prevalence of ROP has increased because of progressive improvement in the survival of preterm infants. The overall incidence of ROP has been reported to be $66 \%$ for any severity of ROP, $18 \%$ for moderately severe ROP, and $6 \%$ for severe ROP in preterm infants born weighing $\leq 1,250 \mathrm{~g}$. ${ }^{7}$ Lower gestational age (GA), lower birth weight, and longer oxygen supply in the postnatal period are known major risk factors for the development of ROP. ${ }^{8}$ Recent studies have suggested an association between chorioamnionitis and ROP. ${ }^{8,9}$ They reported that the incidence of ROP has increased more in infants born to mothers with histologic chorioamnionitis than in infants born to mothers without chorioamnionitis. ${ }^{1,8,9}$ An exposure of the immature retina to changing levels of oxygen leading to dysregulated expression of 
retinal growth factors such as vascular endothelial growth factor (VEGF) and insulin-like growth factor (IGF) seems to play a major role in the development of ROP. ${ }^{9}$ Maternal systemic inflammation, as in acute histologic chorioamnionitis, affects the development of ROP by decreasing levels of IGF-1, ${ }^{9,10}$ which causes abnormal vascularization of the retina. In view of the relationship between acute chorioamnionitis and ROP, we postulated that CPI representing maternal antifetal rejection with derangement in antiangiogenic chemokines can be associated with the development of ROP. This study was conducted to determine if there is a relationship between CPI and ROP in very low-birth-weight (VLBW) infants who were born less than 1,500 g.

\section{MATERIALS AND METHODS}

\section{Study population}

This was a retrospective cohort study of VLBW infants born at Asan Medical Center Children's Hospital, a tertiary academic center, and admitted to neonatal intensive care unit between January 2013 and December 2016. Infants with major congenital malformations or chromosomal abnormalities who died within 72 hours after birth, transferred to other hospitals, or did not have placental biopsies were excluded from analysis (Fig. 1).

\section{Study design}

Perinatal and neonatal clinicopathologic data on each infant including placental biopsies and presence of ROP were retrieved from the electronic medical records. The Institutional Review Board of Asan Medical Center (2017-1058) approved the collec- tion and use of the clinical information for research purposes before the investigation was started and waived the requirement for informed consent.

\section{Placental pathology}

Placental examinations were routinely performed after delivery of VLBW infants. Hematoxylin and eosin-stained sections of the amnion, choriodecidua, umbilical cord, and chorionic plate were examined by pathologists at the same hospital. Histopathological screening of the placenta was performed according to the diagnostic criteria recommend by the Perinatal Section of the Society for Pediatric Pathology. ${ }^{11}$ The placental pathological findings were grouped into four categories: amniotic fluid infection/ inflammation (ACA), maternal vascular underperfusion (MVU), fetal vascular thrombo-occlusive disease (FVTOD), and CPI. The subtypes of CPI included chronic chorioamnionitis (CCA), chronic deciduitis, and villitis of unknown etiology (VUE). ${ }^{12,13}$

\section{Examination and treatment of retinopathy of prematurity}

We had been performing routine eye examinations of neonates whose GA was $<32$ weeks or whose birth weight was $<1,800 \mathrm{~g}$. Eye examinations were performed at postnatal day 28 or at 32 weeks in postmenstrual age by an ophthalmologist. Performance of follow-up eye examinations depended on the disease severity. Diagnoses of ROP were based on the international classification. ${ }^{14}$ Laser treatment was performed for eyes reaching type 1 ROP. Type 1 ROP was divided into zone I, any stage ROP with plus disease; zone I, stage 3 ROP without plus disease; and zone II, stage 2 or 3 ROP with plus disease. ${ }^{15}$ We also included se-

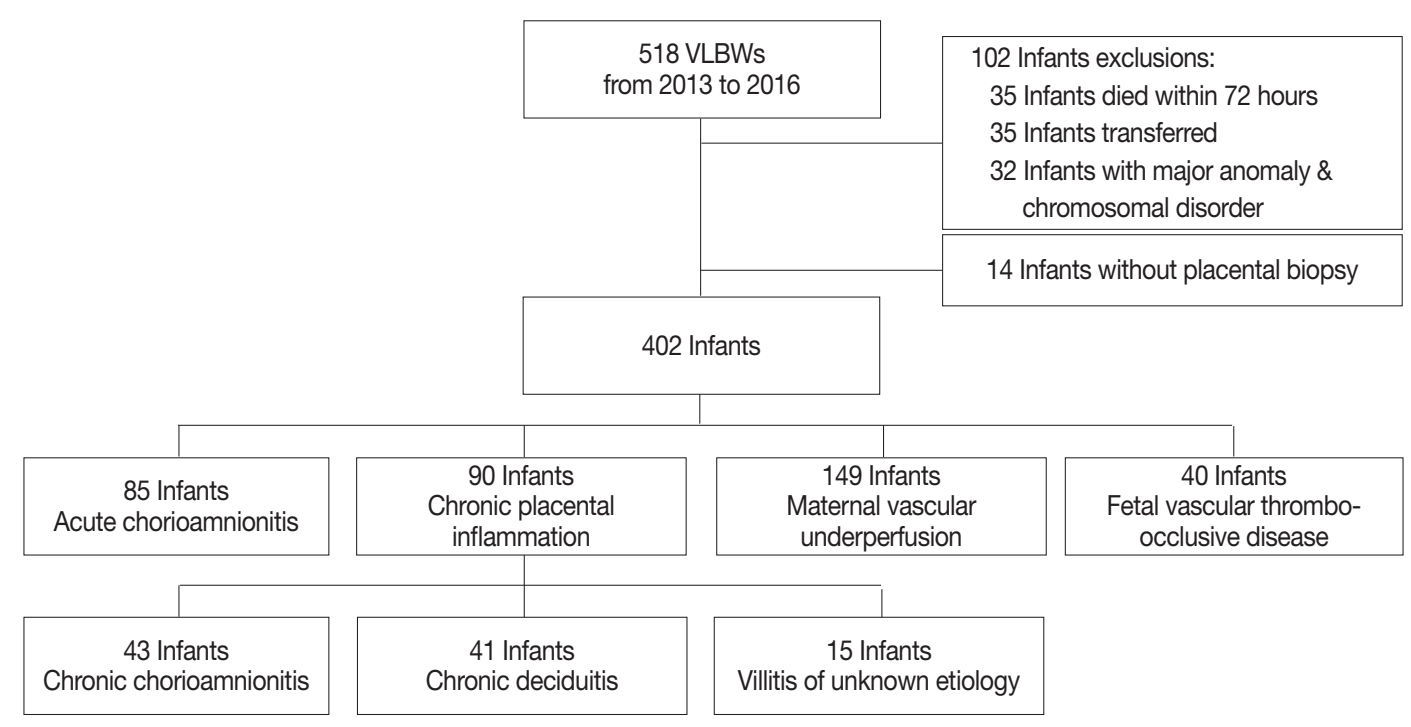

Fig. 1. Study population. VLBW, very low-birth-weight. 
vere ROP as type 1 ROP that required laser photocoagulation.

\section{Clinical data on neonates}

Demographic characteristics such as GA, birth weight, sex, history of multiple births, incidence of maternal preeclampsia, type of delivery, antenatal steroid use, Apgar scores at 1 and 5 minutes, presence of major morbidities such as RDS, patent ductus arteriosus (PDA), BPD, pulmonary arterial hypertension (PAH), significant neurological injury (SNI), necrotizing enterocolitis (NEC), and late-onset sepsis (LOS), duration of hospital stay, and mortality were obtained for each subject. Significant PDA was defined as a condition requiring pharmacological and/or surgical treatment for the mitigation of hemodynamic disturbance. $\mathrm{PAH}$ was diagnosed based on the use of medication according to echocardiographic results. SNI included IVH severer than grade III or periventricular leukomalacia. LOS was defined as bacterial infection confirmed by a positive blood or cerebrospinal fluid culture after postnatal day 7 .

\section{Statistical analysis}

Statistical analysis was performed using SPSS ver. 23.0 (IBM Corp, Armonk, NY, USA). Correlations between CPI and clinical outcomes were determined by performing the chi-square test or Fisher exact test for categorical data and the $t$ test for continuous data. Logistic regression analysis was performed to estimate the independent effects of the main explanatory variables of interest. Statistical significance was defined as $\mathrm{p}<.05$.

\section{RESULTS}

\section{Clinical characteristics of the study population}

A total of 518 VLBW infants were eligible for the study. Of these 518 infants, 35 infants who died within 72 hours, 35 infants who were transferred to other hospitals, 32 infants with major anomalies and chromosomal disorders, and 14 infants without placental biopsies were excluded from analysis (Fig. 1). A total of $402 \mathrm{VLBW}$ infants were included in the analysis. The mean (standard deviation) GA, birth weight, and percentage of males were 28.5 (2.8) weeks, 1,027.2 (304.4) g, and 51.2\%, respectively. The mean maternal age was 33.6 (17.1) years. The incidence of preeclampsia and intrauterine growth retardation (IUGR) were $16.9 \%$ and $33.1 \%$, respectively. Regarding neonatal morbidity, the incidence of RDS, significant PDA, moderate to severe BPD, PAH, SNI, NEC $\geq$ stage 2 , and LOS was $59.2 \%$, $41.5 \%$, $26.1 \%, 3.0 \%, 7.8 \%, 4.7 \%$, and $21.9 \%$, respectively. The incidence of any stage ROP and type 1 ROP requiring laser photo- coagulation was $24.1 \%$ and $11.4 \%$, respectively (Table 1 ). The mean duration of hospital stay of the infants was 67.4 (35.0) days, and the mortality rate was 3.9\%.

\section{Placental pathology}

Of the cases included in this study, 122 did not show any placental pathological finding. Among the remaining 280 cases, 85 cases showed findings consistent with ACA, 90 cases showed CPI, 149 cases showed MVU, and 40 cases showed FVTOD (Fig. 1). Two or more placental pathological findings were superimposed in 82 cases, and one placental pathological finding was superimposed in 198 cases (Fig. 2). Of the 90 cases with CPI, 43 were with CCA, 41 were with chronic deciduitis, 15 were with VUE, and 16 showed overlapping patterns (Fig. 1).

Table 1. Baseline characteristics and morbidity of the study cohort

\begin{tabular}{lc}
\hline Variable & Value $(\mathrm{n}=402)$ \\
\hline Neonatal characteristics & \\
Gestational age (wk) & $28.5 \pm 2.8$ \\
Birth weight (g) & $1,027.2 \pm 304.4$ \\
Male & $206(51.2)$ \\
Multiple births & $94(23.4)$ \\
Maternal age (yr) & $33.6 \pm 17.1$ \\
Pre-eclampsia & $69(16.9)$ \\
Cesarean section & $305(75.1)$ \\
Antenatal steroid use & $363(90.3)$ \\
Apgar score at 1 min & $5.0(3-7)$ \\
Apgar score at 5 min & $7.0(1-9)$ \\
IUGR & $133(33.1)$ \\
Neonatal morbidity & \\
RDS & $238(59.2)$ \\
Significant PDA & $167(41.5)$ \\
Moderate to severe BPD & $105(26.1)$ \\
Postnatal steroid therapy & $96(23.9)$ \\
Duration of oxygen supply (day) & $40.4 \pm 2.1$ \\
Pulmonary hypertension & $12(3.0)$ \\
Severe neurologic injury (IVH $\geq$ grade 3 or PVL) & $43(7.8)$ \\
NEC ( $\geq$ stage 2) & $19(4.7)$ \\
ROP (any stage) & $97(24.1)$ \\
Type 1 ROP & $46(11.4)$ \\
Anti-VEGF therapy & $14(2.7)$ \\
LOS & $88(21.9)$ \\
Hospital stay (day) & $67.4 \pm 35.0$ \\
Mortality & $16(3.9)$ \\
\hline & \\
\hline
\end{tabular}

Values are presented as mean $\pm \mathrm{SD}$, number (\%), or mean (IQR). IUGR, intrauterine growth retardation; RDS, respiratory distress syndrome; PDA, patent ductus arteriosus; BPD, bronchopulmonary dysplasia; IVH, intraventricular hemorrhage; PVL, periventricular leukomalacia; NEC, necrotizing enterocolitis; ROP, retinopathy of prematurity; VEGF, vascular endothelial growth factor; LOS, late-onset sepsis; SD, standard deviation; IQR, interquartile range. 

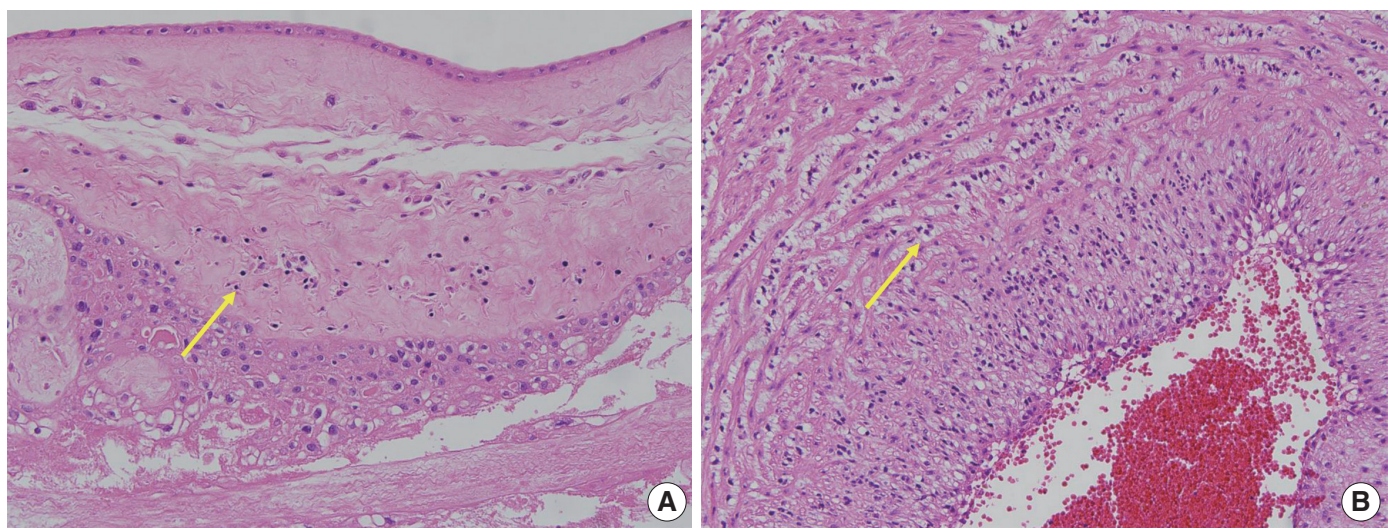

Fig. 2. Microscopic features of chronic chorioamnionitis and acute funisitis (representative case). (A) Lymphocytes (arrow) infiltrate into the chorionic trophoblast layer of the chorioamniotic membrane. (B) Neutrophils (arrow) infiltrate into the smooth muscle layer of the umbilical artery.

Table 2. Characteristics of infants on the basis of CPI

\begin{tabular}{|c|c|c|c|}
\hline Variable & Non-CPI $(n=312)$ & $\mathrm{CPI}(n=90)$ & $p$-value \\
\hline Gestational age (wk) & $29.1 \pm 2.9$ & $28.4 \pm 3.2$ & $.007^{*}$ \\
\hline Birth weight (g) & $1,064.7 \pm 286.9$ & $1,012.5 \pm 309.1$ & .589 \\
\hline Sex & & & .506 \\
\hline Male & $154(49.3)$ & $48(53.3)$ & \\
\hline Female & $158(50.6)$ & $42(46.7)$ & \\
\hline Pre-eclampsia & $78(25.0)$ & $9(10.0)$ & $.031^{*}$ \\
\hline Antenatal steroid use & $284(91.0)$ & $79(87.8)$ & .398 \\
\hline IUGR & $107(34.3)$ & $26(28.9)$ & .338 \\
\hline RDS & $177(56.7)$ & $61(67.8)$ & .068 \\
\hline Significant PDA & $127(40.7)$ & $40(44.4)$ & .531 \\
\hline Moderate to severe BPD & 79 (25.3) & $26(28.9)$ & .497 \\
\hline Postnatal steroid therapy & $72(23.1)$ & $24(26.7)$ & .496 \\
\hline Duration of oxygen supply (day) & $41.4 \pm 38.2$ & $41.0 \pm 38.1$ & .163 \\
\hline Severe neurologic injury (IVH $\geq$ grade 3 or $\mathrm{PVL}$ ) & $34(10.9)$ & $9(10)$ & .051 \\
\hline NEC ( $\geq$ stage 2 ) & $15(4.8)$ & $4(4.4)$ & .882 \\
\hline LOS & $65(20.8)$ & 23 (25.6) & .164 \\
\hline ROP (any stage) & $71(22.8)$ & $26(28.9)$ & $.021^{*}$ \\
\hline Type 1 ROP & $27(8.6)$ & $19(21.1)$ & $.003^{*}$ \\
\hline Hospital days (day) & $66.5 \pm 36.0$ & $72.6 \pm 36.1$ & .154 \\
\hline
\end{tabular}

Values are presented as mean \pm SD or number (\%).

CPI, chronic placental inflammation; IUGR, intrauterine growth retardation; RDS, respiratory distress syndrome; PDA, patent ductus arteriosus; BPD, bronchopulmonary dysplasia; IVH, intraventricular hemorrhage; PVL, periventricular leukomalacia; NEC, necrotizing enterocolitis; LOS, late-onset sepsis; ROP, retinopathy of prematurity; SD, standard deviation.

${ }^{*} \mathrm{p}<.05$.

\section{Association of $\mathrm{CPI}$ and retinopathy of prematurity}

Of the 90 cases with CPI, $28.9 \%$ (26 of 90) developed any stage ROP, and $21.1 \%$ (19 of 90) underwent laser photocoagulation (Table 2). The infants with CPI showed a significantly lower $\mathrm{GA}(\mathrm{p}=.007)$ and had a higher incidence of any stage ROP and history of laser photocoagulation treatment than the infants without CPI did ( $\mathrm{p}=.021$ and $\mathrm{p}=.003)$. Other characteristics were not significantly different between the two groups (Table 2).

Lower GA, lower birth weight, severe BPD, and longer dura- tion of oxygenation treatment were associated with greater increases in the incidence of type 1 ROP. According to the pathological findings of the placenta, CPI and ACA statistically correlated with any stage ROP (odds ratio [OR], 2.050; 95\% confidence interval [CI], 1.170 to $3.592 ; \mathrm{p}=.038$ and $\mathrm{OR}, 2.079 ; 95 \% \mathrm{CI}$, 1.177 to $3.671 ; \mathrm{p}=.012$ ) and type 1 ROP for laser photocoagulation (OR, 2.825; 95\% CI, 1.487 to 5.367; $\mathrm{p}=.002$ and $\mathrm{OR}$, $1.977 ; 95 \% \mathrm{CI}, 1.012$ to $3.862 ; \mathrm{p}=.046$ ) in univariate analysis (Table 3). 
Table 3. Logistic regression analysis to identify risk factors for any stage ROP and type 1 ROP

\begin{tabular}{|c|c|c|c|c|c|c|c|c|}
\hline \multirow[b]{2}{*}{ Variable } & \multicolumn{4}{|c|}{ Any stage ROP } & \multicolumn{4}{|c|}{ Type 1 ROP } \\
\hline & $\begin{array}{l}\text { No ROP } \\
(n=305)\end{array}$ & $\begin{array}{c}\mathrm{ROP} \\
(\mathrm{n}=97)\end{array}$ & $\begin{array}{c}\mathrm{OR} \\
(95 \% \mathrm{Cl})\end{array}$ & $p$-value & $\begin{array}{c}\text { No type } 1 \\
\text { ROP }(n=356)\end{array}$ & $\begin{array}{c}\text { Type 1 ROP } \\
(n=46)\end{array}$ & OR $(95 \% \mathrm{Cl})$ & $p$-value \\
\hline Gestational age (wk) & $30.0 \pm 2.5$ & $25.7 \pm 1.7$ & $0.872(0.847-0.897)$ & $<.001^{*}$ & $30.4 \pm 2.8$ & $25.1 \pm 1.5$ & $0.882(0.853-0.911)$ & $<.001^{*}$ \\
\hline Birth weight (g) & $1,152.5 \pm 239.5$ & $740.6 \pm 212.9$ & $0.993(0.992-0.995)$ & $<.001^{*}$ & $1100 \pm 267.7$ & $7680.8 \pm 198.7$ & $0.993(0.991-0.995)$ & $<.001^{*}$ \\
\hline Male & $157(51.5)$ & $49(50.5)$ & $1.000(0.329-1.126)$ & .339 & $179(50.3)$ & $23(50.0)$ & $1.000(0.504-1.305)$ & .219 \\
\hline Pre-eclampsia & $62(20.3)$ & $7(7.2)$ & $0.919(0.383-2.205)$ & .851 & $66(18.5)$ & $3(6.5)$ & $0.295(0.089-0.981)$ & $.046^{*}$ \\
\hline Antenatal steroid & $271(88.9)$ & $92(94.8)$ & $1.547(0.484-4.942)$ & .461 & $321(90.2)$ & $42(91.3)$ & $1.145(0.388-3.382)$ & .807 \\
\hline IUGR & $114(37.3)$ & $19(19.6)$ & $0.369(0.323-1.265)$ & .199 & $125(35.1)$ & $8(17.4)$ & $0.389(0.176-0.860)$ & $.020^{*}$ \\
\hline Moderate to severe BPD & $40(13.1)$ & $65(67.0)$ & $0.503(0.361-0.508)$ & $<.001^{*}$ & $67(18.8)$ & 38 (82.6) & $0.467(0.281-0.410)$ & $<.001^{*}$ \\
\hline Postnatal steroid therapy & $34(11.1)$ & $62(63.9)$ & $0.512(0.380-0.531)$ & $<.001^{*}$ & $60(16.8)$ & $36(78.3)$ & $0.399(0.382-0.606)$ & $<.001^{*}$ \\
\hline Duration of oxygen treatment (day) & $27.9 \pm 26.1$ & $81.2 \pm 42.7$ & $1.054(1.042-1.066)$ & $<.001^{*}$ & $33.5 \pm 31.3$ & $98.6 \pm 38.4$ & $1.044(1.031-1.057)$ & $<.001^{*}$ \\
\hline \multicolumn{9}{|l|}{ Placental pathology } \\
\hline $\mathrm{CPI}$ & $64(21.0)$ & $26(26.8)$ & $2.050(1.170-3.592)$ & $.038^{*}$ & $71(19.9)$ & $19(46.3)$ & $2.825(1.487-5.367)$ & $.002^{*}$ \\
\hline CCA & $33(10.8)$ & $10(10.3)$ & $0.159(0.691-3.152)$ & .159 & $36(10.1)$ & $7(15.2)$ & $1.590(0.663-3.816)$ & .299 \\
\hline Chronic deciduitis & $29(9.5)$ & $12(12.4)$ & $0.244(0.874-3.872)$ & .244 & $33(9.3)$ & $8(17.4)$ & $2.061(0.887-4.784)$ & .093 \\
\hline VUE & $13(4.3)$ & $2(2.1)$ & $0.139(0.042-2.501)$ & .139 & $15(4.2)$ & 0 & $0.237(0.013-4.413)$ & .335 \\
\hline $\mathrm{ACA}$ & $53(17.4)$ & $32(33.0)$ & $2.079(1.177-3.671)$ & $.012^{*}$ & $70(19.7)$ & $15(32.6)$ & $1.977(1.012-3.862)$ & $.046^{*}$ \\
\hline MVU & $118(38.7)$ & $31(32.0)$ & $0.744(0.452-1.344)$ & .744 & $134(37.6)$ & $15(32.6)$ & $0.802(0.417-1.540)$ & .507 \\
\hline FVTOD & $29(9.5)$ & $11(11.3)$ & $0.806(0.325-2.000)$ & .642 & $36(10.1)$ & $4(8.7)$ & $0.847(0.287-2.498)$ & .763 \\
\hline
\end{tabular}

Values are presented as mean \pm SD or number $(\%)$

ROP, retinopathy of prematurity; OR, odds ratio; Cl, confidence interval; IUGR, intrauterine growth retardation; BPD, bronchopulmonary dysplasia; CPI, chronic placental inflammation; CCA, chronic chorioamnionitis; VUE, villitis of unknown etiology; ACA, amniotic fluid infection/inflammation; MVU, maternal vascular underperfusion; FVTOD, fetal vascular thrombo-occlusive disease; SD, standard deviation. ${ }^{*} p<.05$.

Table 4. Placental histology associated with any stage ROP and type 1 ROP

\begin{tabular}{|c|c|c|c|c|c|c|}
\hline \multirow{2}{*}{$\begin{array}{l}\text { Placental } \\
\text { pathology }\end{array}$} & \multicolumn{2}{|l|}{ No ROP } & \multicolumn{2}{|c|}{ Any stage ROP } & \multicolumn{2}{|c|}{ Type 1 ROP } \\
\hline & Adjusted OR (95\% Cl) & $p$-value ${ }^{a}$ & Adjusted OR (95\% Cl) & $p$-value ${ }^{a}$ & Adjusted OR (95\% Cl) & $p$-value ${ }^{a}$ \\
\hline $\mathrm{CPI}$ & $1.008(0.492-2.068)$ & .982 & $1.647(0.702-3.992)$ & .246 & 2.739 (1.112-6.749) & $.029^{*}$ \\
\hline ACA & $0.811(0.429-1.940)$ & .811 & $1.267(0.531-3.023)$ & .594 & $0.833(0.319-2.173)$ & .708 \\
\hline MVU & $0.630(0.315-1.261)$ & .630 & 2.036 (0.865-4.792) & .103 & $0.570(0.211-1.539)$ & .267 \\
\hline FVTOD & $0.815(0.298-2.226)$ & .690 & $1.080(0.360-3.240)$ & .126 & $0.424(0.141-1.272)$ & .126 \\
\hline
\end{tabular}

Chronic placental inflammation was statistically associated with type $1 \mathrm{ROP}$ by multiple logistic regression analysis.

$\mathrm{ROP}$, retinopathy of prematurity; OR, odds ratio; $\mathrm{Cl}$, confidence interval; $\mathrm{CPI}$, chronic placental inflammation; $\mathrm{ACA}$, amniotic fluid infection/inflammation; MVU, maternal vascular underperfusion; FVTOD, fetal vascular thrombo-occlusive disease.

${ }^{*} \mathrm{p}<.05$.

${ }^{a}$ Adjusted for gestational age, birth weight, sex, duration of oxygen treatment, pre-eclampsia, IUGE and the overlapping placental pathology.

Table 4 compares the placental pathological findings in terms of their effect on any stage ROP and type 1 ROP. Multiple logistic regression analysis adjusted for $\mathrm{GA}$, birth weight, sex, incidence of preeclampsia and IUGR, duration of oxygen supply, and overlapping placental pathology was performed. CPI was found to be independently associated with type 1 ROP (adjusted OR, $2.739 ; 95 \% \mathrm{CI}, 1.112$ to $6.749 ; \mathrm{p}=.029$ ). ACA was significantly associated with type $1 \mathrm{ROP}$ in the univariate analysis, but not with type 1 ROP after adjustment analysis. Any stage ROP was not significantly associated with any placental finding after adjustment analysis.

\section{DISCUSSION}

Many investigators have studied the relationship between placental lesions in VLBW infants and clinical complications because examinations frequently provide information valuable for the management of neonates. ${ }^{2,4-6}$ For example, the presence of acute chorioamnionitis and funisitis indicates in utero fetal systemic inflammation as a consequence of ACA. ${ }^{16}$ In this study, we showed a significant correlation between CPI and severe ROP that required laser photocoagulation (type 1 ROP) in VLBW infants for the first time.

A prominent cause of visual impairment and blindness in children, ROP is a developmental perturbation of the retina., ${ }^{9,17,18}$ 
The structural characteristics of ROP include the initial cessation of retinal vascularization and subsequent proliferation of retinal vessels. ${ }^{17,19}$ ROP is caused by impaired autoregulation of the retinal blood vessels and sudden postnatal oxygen tension. Retinal neovascularization in ROP cases involves angiogenic factors such as VEGF in preterm infants and VLBW infants. ${ }^{20-22}$ Recent studies have reported that lower GA, lower birth weight, and excessive oxygen exposure are major risk factors for ROP. ${ }^{20-23}$ Other studies demonstrated that both antenatal and neonatal exposures to inflammation are consistently associated with an increased risk of ROP as well as cerebral white matter damage and BPD in preterm infants. ${ }^{9,24,25}$ The formation of new blood vessels from existing vessels, angiogenesis, is affected not only by normal physiological growth, but also by pathological events such as inflammation. ${ }^{24,26}$ Inflammation is a host defense mechanism serving to protect tissues from infection and injury, and studies have shown that proinflammatory cytokines such as tumor necrosis factor $\alpha$ and IL-1 function as proangiogenic factors. ${ }^{24,27-29}$

Although oxygen supply is far better managed by using noninvasive ventilators today, ROP remains a major issue because survival rates of infants with extremely low GA and birth weight and immature retinas at high risk of ROP have substantially increased. The lack of in utero angiogenic factors such as VEGF, IGF-1, and erythropoietin significantly increases the risk of ROP. $^{20,30}$ Omega long-chain polyunsaturated fatty acids, which are provided by maternal and fetal interaction, are also important for retinal development. ${ }^{30-32}$ Therefore, the risk of ROP may increase in cases of intrauterine insufficiency of angiogenic factors in infants with lower GA and birth weight.

It has been recently proposed that idiopathic CPI is a feature of maternal antifetal rejection mimicking allograft rejection. ${ }^{12}$ The placenta and fetus are semiallografts to the mother, and fetal (paternal) human leukocyte antigen-specific antibodies are detected in the sera of subsets of pregnant women. ${ }^{6,12} \mathrm{CPI}$ is characterized by the infiltration of maternal $\mathrm{CD}^{+} \mathrm{T}$ cells into fetal placental tissues and associated with the overexpression of $\mathrm{C}-\mathrm{X}-\mathrm{C}$-motif chemokines such as CXCL9, CXCL10, and CXCL11 in placental tissues. ${ }^{6,12}$ Also a marker of CPI coupled with maternal-fetal rejection, ${ }^{33,34}$ elevation of CXCL10 level has been shown to occur in the event of rejection of allografts such as the liver and kidneys. ${ }^{35}$

The findings in this study suggest that CPI may be at least partially responsible for the increased incidence of severe ROP that required laser photocoagulation after adjustment for GA, birth weight, sex, duration of oxygen supply, and other overlapping placental pathologies. We did not adjust for BPD because the condition is a confounding variable overlapping with the duration of oxygen supply. The association between CPI and severe ROP suggests that maternal-fetal rejection associated with increased fetal plasma antiangiogenic CXCL10 level contributes to abnormal angiogenesis of the retina in VLBW infants. A relationship between the subtypes of ROP and the intrauterine environment that triggers preterm birth also has been proposed. ${ }^{36}$ Likewise, acute chorioamnionitis may increase the risk of ROP by directly sensitizing the developing retina to oxygen-induced changes in VEGF availability and subsequent vascular development or by causing systemic hypotension resulting in retinal hypoperfusion or ischemia. ${ }^{8,9,25}$

Decreased production of placental growth factors (PGF), 45to $50-\mathrm{kDa}$ dimeric glycoproteins with $53 \%$ sequence homology with VEGF, plays a key role in placental angiogenesis. ${ }^{37-40}$ PGF may have therapeutic potential in cases of disorders associated with problems of angiogenesis such as cancer, retinopathy, and certain inflammatory disorders. ${ }^{40}$ This is another piece of evidence indicating the importance of placenta-derived growth factors for developmental angiogenesis. Therefore, it is plausible that CPImediating antiangiogenic effects lead to deregulated angiogenesis of ROP in VLBW infants.

There are some limitations of our study. First, it was a retrospective study of a relatively small number of inborn infants at a single hospital; thus, the findings might not extrapolate to a larger population. Second, multiple pathological diagnoses were given in some cases, which can interfere with the strength of the association. Third, in the association between CPI and type 1 ROP, we had a wide confidence interval (1.112-6.749) because many factors may play a contributory role in ROP development. Despite these limitations, this study demonstrates an association between CPI and severe ROP in VLBW infants. Future studies that examine the direct causality of CPI in the development of retinal damage and ROP are needed.

In conclusion, the pathological findings of the placenta are important in predicting the outcomes of preterm infants and VLBW infants. This study shows for the first time that CPI may affect the development of type 1 ROP requiring treatment of laser photocoagulation in VLBW infants.

\section{ORCID}

Chae Young Kim: https://orcid.org/0000-0002-9258-7499

Euiseok Jung: https://orcid.org/0000-0003-0693-5627

Eun Na Kim: https://orcid.org/0000-0003-2992-7881

Chong Jai Kim: https://orcid.org/0000-0002-2844-9446

Joo Yong Lee: https://orcid.org/0000-0002-2187-196X 
Ji Hye Hwang: https://orcid.org/0000-0003-1080-1728

Woo Sun Song: https://orcid.org/0000-0002-2786-9836

Byong Sop Lee: https://orcid.org/0000-0002-1347-4200

Ellen Ai-Rhan Kim: https://orcid.org/0000-0002-9859-3021

Ki-Soo Kim: https://orcid.org/0000-0003-1547-5220

\section{Conflicts of Interest}

No potential conflict of interest relevant to this article was reported.

\section{REFERENCES}

1. Galinsky R, Polglase GR, Hooper SB, Black MJ, Moss TJ. The consequences of chorioamnionitis: preterm birth and effects on development. J Pregnancy 2013; 2013: 412831.

2. Morgan TK. Role of the placenta in preterm birth: a review. Am J Perinatol 2016; 33: 258-66.

3. Redline RW. Placental pathology: a systematic approach with clinical correlations. Placenta 2008; 29 Suppl A: S86-91.

4. Lee J, Kim JS, Park JW, et al. Chronic chorioamnionitis is the most common placental lesion in late preterm birth. Placenta 2013; 34: 681-9.

5. Kim CJ, Romero R, Chaemsaithong P, Chaiyasit N, Yoon BH, Kim YM. Acute chorioamnionitis and funisitis: definition, pathologic features, and clinical significance. Am J Obstet Gynecol 2015; 213(4 Suppl): S29-52.

6. Kim CJ, Romero R, Kusanovic JP, et al. The frequency, clinical significance, and pathological features of chronic chorioamnionitis: a lesion associated with spontaneous preterm birth. Mod Pathol 2010; 23: 1000-11.

7. Martin RJ, Fanaroff AA, Walsh MC. Fanaroff and Martin's neonatal-perinatal medicine. 10th ed. Philadelphia: Elsevier Health Sciences, 2014.

8. Liu PM, Fang PC, Huang CB, et al. Risk factors of retinopathy of prematurity in premature infants weighing less than $1600 \mathrm{~g}$. Am J Perinatol 2005; 22: 115-20.

9. Lee J, Dammann O. Perinatal infection, inflammation, and retinopathy of prematurity. Semin Fetal Neonatal Med 2012; 17: 26-9.

10. Woo SJ, Park KH, Jung HJ, et al. Effects of maternal and placental inflammation on retinopathy of prematurity. Graefes Arch Clin Exp Ophthalmol 2012; 250: 915-23.

11. Redline RW, Heller D, Keating S, Kingdom J. Placental diagnostic criteria and clinical correlation: a workshop report. Placenta 2005; 26 Suppl A: S114-7.

12. Kim CJ, Romero R, Chaemsaithong P, Kim JS. Chronic inflammation of the placenta: definition, classification, pathogenesis, and clinical significance. Am J Obstet Gynecol 2015; 213(4 Suppl): S53-69.

13. Redline RW. Classification of placental lesions. Am J Obstet Gynecol 2015; 213(4 Suppl): S21-8.

14. International Committee for the Classification of Retinopathy of Prematurity. The International Classification of Retinopathy of Prematurity revisited. Arch Ophthalmol 2005; 123: 991-9.

15. Early Treatment for Retinopathy of Prematurity Cooperative Group. Revised indications for the treatment of retinopathy of prematurity: results of the early treatment for retinopathy of prematurity randomized trial. Arch Ophthalmol 2003; 121: 1684-94.

16. Romero R, Salafia CM, Athanassiadis AP, et al. The relationship between acute inflammatory lesions of the preterm placenta and amniotic fluid microbiology. Am J Obstet Gynecol 1992; 166: 1382-8.

17. Mechoulam H, Pierce EA. Retinopathy of prematurity: molecular pathology and therapeutic strategies. Am J Pharmacogenomics 2003; 3: 261-77.

18. Hansen RM, Moskowitz A, Akula JD, Fulton AB. The neural retina in retinopathy of prematurity. Prog Retin Eye Res 2017; 56: 32-57.

19. Smith LE. Pathogenesis of retinopathy of prematurity. Growth Horm IGF Res 2004; 14 Suppl A: S140-4.

20. Chen J, Smith LE. Retinopathy of prematurity. Angiogenesis 2007; 10: $133-40$.

21. Tasman W, Patz A, McNamara JA, Kaiser RS, Trese MT, Smith BT. Retinopathy of prematurity: the life of a lifetime disease. Am J Ophthalmol 2006; 141: 167-74.

22. Hartnett ME. Role of cytokines and treatment algorithms in retinopathy of prematurity. Curr Opin Ophthalmol 2017; 28: 282-8.

23. Flynn JT, Bancalari E, Snyder ES, et al. A cohort study of transcutaneous oxygen tension and the incidence and severity of retinopathy of prematurity. N Engl J Med 1992; 326: 1050-4.

24. Dammann O, Brinkhaus MJ, Bartels DB, et al. Immaturity, perinatal inflammation, and retinopathy of prematurity: a multi-hit hypothesis. Early Hum Dev 2009; 85: 325-9.

25. Sood BG, Madan A, Saha S, et al. Perinatal systemic inflammatory response syndrome and retinopathy of prematurity. Pediatr Res 2010; 67: 394-400.

26. Kobayashi H, Lin PC. Angiogenesis links chronic inflammation with cancer. Methods Mol Biol 2009; 511: 185-91.

27. Folkman J, Shing Y. Angiogenesis. J Biol Chem 1992; 267: 10931-4.

28. Voronov E, Shouval DS, Krelin Y, et al. IL-1 is required for tumor invasiveness and angiogenesis. Proc Natl Acad Sci U S A 2003; 100: 2645-50.

29. Costa C, Incio J, Soares R. Angiogenesis and chronic inflammation: cause or consequence? Angiogenesis 2007; 10: 149-66.

30. Hellström A, Engström E, Hård AL, et al. Postnatal serum insulinlike growth factor I deficiency is associated with retinopathy of 
prematurity and other complications of premature birth. Pediatrics 2003; 112: 1016-20.

31. Connor KM, SanGiovanni JP, Lofqvist C, et al. Increased dietary intake of omega-3-polyunsaturated fatty acids reduces pathological retinal angiogenesis. Nat Med 2007; 13: 868-73.

32. Najm S, Löfqvist C, Hellgren G, et al. Effects of a lipid emulsion containing fish oil on polyunsaturated fatty acid profiles, growth and morbidities in extremely premature infants: A randomized controlled trial. Clin Nutr ESPEN 2017; 20: 17-23.

33. Romero R, Chaemsaithong P, Chaiyasit N, et al. CXCL10 and IL-6: Markers of two different forms of intra-amniotic inflammation in preterm labor. Am J Reprod Immunol 2017; 78: e12685.

34. Romagnani P, Lasagni L, Annunziato F, Serio M, Romagnani S. CXC chemokines: the regulatory link between inflammation and angiogenesis. Trends Immunol 2004; 25: 201-9.

35. Lazzeri E, Rotondi M, Mazzinghi B, et al. High CXCL10 expression in rejected kidneys and predictive role of pretransplant serum CXCL10 for acute rejection and chronic allograft nephropathy.
Transplantation 2005; 79: 1215-20.

36. Lynch AM, Wagner BD, Hodges JK, et al. The relationship of the subtypes of preterm birth with retinopathy of prematurity. Am J Obstet Gynecol 2017; 217: 354.e1-e8.

37. Powe CE, Levine RJ, Karumanchi SA. Preeclampsia, a disease of the maternal endothelium: the role of antiangiogenic factors and implications for later cardiovascular disease. Circulation 2011; 123: 2856-69.

38. Taylor RN, Grimwood J, Taylor RS, McMaster MT, Fisher SJ, North RA. Longitudinal serum concentrations of placental growth factor: evidence for abnormal placental angiogenesis in pathologic pregnancies. Am J Obstet Gynecol 2003; 188: 177-82.

39. Zhu T, Zhang L, Zhao F, Qu Y, Mu D. Association of maternal hypertensive disorders with retinopathy of prematurity: a systematic review and meta-analysis. PLoS One 2017; 12: e0175374.

40. Luttun A, Tjwa M, Carmeliet P. Placental growth factor (PIGF) and its receptor Flt-1 (VEGFR-1): novel therapeutic targets for angiogenic disorders. Ann NY Acad Sci 2002; 979: 80-93. 